Apidologie, 1975, 6 (3), 283-293.

\title{
DIE FEUCHTIGKEIT DER STOCKLUFT UND DIE BRUTTÄTIGKEIT DER BIENEN (APIS MELLIFICA L.) IN EINEM FLUGRAUM ${ }^{1} 2$
}

\author{
L'humidité de l'air dans la ruche \\ et la production de couvain chez l'abeille (Apis mellifica L.) \\ dans une chambre de vol
}

J. P. van PRAAGH

Lab. für vergleichende Physiologie

Rijkuniversiteit Utrecht

\begin{abstract}
SUMMARY
HUMIDITY INSIDE THE HIVE AND BROOD REARING BY THE HONEYBEE (Apis mellifica L.) in A FLIGHT RоOм
\end{abstract}

A system which enables dew-point-measurements between combs in a occupied hive is described. The results of dew-point-measurement at four sites in a beehive are given.

The colony stood in a bee flight room. The role of air humidity for brood rearing is examined. Following results are obtained.

1. A colony in a flight room is unable to keep up the air humidity inside the hive.

2. A dry air inside makes the brood rearing activity decreasing.

3. The from nectar evaporated amount of water plays an important part in the water vapour economy of the colony.

4. A large amount of water is gathered when we offer pure honey to gather. The water gathering behaviour is not triggered by dry air outside the hive which leads towards water vapour losses of the air inside the hive when we offer nectar (45\% sugar). As a result the air humidity inside becomes too low for normal brood rearing activity.

1. Diese Arbeit wurde von der Niederländischen Organisation für reinwissenschaftliche Forschung (Z.W.O.) gefördert.

2. Herrn Prof. Dr. Karl von Frisci zum 88. Geburtstag gewidmet. 


\section{ZUSAMMENF ASSUNG}

Mittels eines beschriebenen Taupunktmeßsystems wurde die Luftfeuchtigkeit innerhalb eines Bienenstockes an vier Stellen gleichzeitig bestimmt. Das Volk stand in einem geschlossenen Flugraum. Daneben wurde die Bedeutung der Luftfeuchtigkeit in diesem Flugraum für die Bruttätigkeit untersucht. Es hat sich folgendes ergeben :

1) Ein Volk im Flugraum ist nicht in der Lage, das Austrocknen der Stockluft zu verhindern.

2) Austrocknung der Stockluft schadet der Bruttätigkeit.

3) Das Eindicken von Nektar spielt beim Wasserdampfhaushalt eine wichtige Rolle.

4) Das Wassersammeln wird nur durch Füttern von reinem Honig ( $80 \%$ Zucker) ausgelöst, nicht aber durch grössere Wasserdampfverluste an die Aussenluft bei einem normalen Nektarangebot (45\% Zucker), sodass in diesem Falle die Stockluft austrocknet und der Feuchtigkeitsgrad für die normale Bruttätigkeit zu niedrig wird.

\section{EINLEITUNG}

Dass die Feuchtigkeit der Aussenluft bei der Brutnestentwicklung eines Bienenvolkes im Flugraum vielleicht eine wichtige Rolle spielt, wurde schon in einer früheren Arbeit vermutet (v. PraAgh 1972). Ein Einfluss der Luftfeuchte im Klimaraum auf die Brutnestentwicklung wurde auch von Van LAERE (1963) beobachtet. Bei Van LAERE genügt ein Wasserdampfgehalt von $20 \mathrm{~g} / \mathrm{m}^{3}$ nicht, um die Bruttätigkeit aufrecht zu erhalten. Bei v. PraAgh reicht eine solche Feuchtigkeit für eine stabile Bruttätigkiet.

BüDEL (1948) glaubte an Hand von Meßergebnissen an bewohnten Beuten den Nachweis erbracht zu haben, dass die Bienen imstande sind, den Wasserdampfhaushalt der Beute zu regulieren. Er hat mit einem Aspirationspsychrometer nach Assmann an verschiedenen Stellen 0,5 L Luft aus den Beuten entnommen. BüDEL findet im. Brutnest eine relative Luftfeuchtigkeit zwischen $30 \%$ und $50 \%$. Diese Werte entsprechen bei $34{ }^{\circ} \mathrm{C}$ einem Taupunkt zwischen $14{ }^{\circ} \mathrm{C}$ und $22{ }^{\circ} \mathrm{C}$, einer absoluten Luftfeuchte von $12 \mathrm{~g} / \mathrm{m}^{3}$ und $19,5 \mathrm{~g} / \mathrm{m}^{3}$ und einem Partialdampfdruck zwischen 15,6 mbar und 26,6 mbar $^{3}$.

Die absolute Feuchte der Stockluft im Winter schwankt zwischen $1 \mathrm{~g} / \mathrm{m}^{3}$ und $7 \mathrm{~g} / \mathrm{m}^{3}$.

Die Verhältnisse in der Wintertraube wurden durch Simpson (1950) mittels Taupunktmessungen in situ bestimmt. Seine Angaben sind höher als

3. Begriffserläuterungen :

Die Taupunkttemperatur in ${ }^{\circ} \mathrm{C}$ gibt die Temperatur an, bei der Sättigung der Luft und damit Wasserkondensation eintritt, wenn sich die Luft auf den Wert der Taupunkttemperatur abkühlt.

Die Absolute Lufifeuchte in $\mathrm{g} / \mathrm{m}^{3}$ ist der Wasserdampfgehalt der Luft, sie ist also vollkommen temperaturunabhängig.

Der Partialdampfdruck in mbar ist der Wasserdampfdruck in der Luft als Anteil des barometrischen Druckes der feuchten Luft.

Der Sättigungsdampfdruck in mbar ist der bei der jeweiligen Lufttemperatur maximal mögliche Wasserdampfdruck.

Die Relative Luftfeuchte in Prozent ist das Verhältnis zwischen dem wahren Wasserdampfgehalt in der Luft und dem Sättigungsmaximum bei der jeweiligen Lufttemperatur. 
die oben angegebenen Werte; er fand eine absolute Luftfeuchte ohne Bruttätigkeit zwischen $11,5 \mathrm{~g} / \mathrm{m}^{3}$ und $17 \mathrm{~g} / \mathrm{m}^{3}$ und während der Bruttätigkeit (von Mitte Februar bis Ende April) von $13 \mathrm{~g} / \mathrm{m}^{3}$ bis $18,5 \mathrm{~g} / \mathrm{m}^{3}$.

Über den Zusammenhang zwischen Wasserhaushalt und Temperaturregulierung sind wir weitgehend informiert durch die Arbeit von Lindauer (1954). Die Bienen reagieren auf Überhitzungsgefahr mit Wassereintragen; durch Fächeln wird die Wasserverdunstung und damit die Abkühlung der Stockluft gefördert. Dazu gibt Lindauer an, dass der Wasserbedarf eines Bienenvolkes von der Menge der offenen Brut im Stock abhängig sei. Je mehr Brut, um so höher der Wasserbedarf. Das Nektarangebot spielt dabei auch eine Rolle; bei guter Tracht geht der Wasserbedarf stark zurück; das Volk hat die Möglichkeit, seinen Wasserbedarf aus dem eingetragenen Nektar zu decken. Lindauer vermutet, dass das Rüsselschlagen (das Verdunsten von Wasser) primär gegen eine Überhitzung und nicht gegen die Gefahr einer zu grossen Trockenheit der Stockluft gerichtet ist. Fächeln wird nach Hazelnoff (1941) ausgelöst durch Überhitzung, Erhöhung des $\mathrm{CO}_{2}$-Partialdampfdruckes, Erniedrigung des $\mathrm{O}_{2}$-Partialdampfdruckes. Durch das Fächeln wird 0,5 bis 1,0 L Luft pro Sekunde von oben nach unten und durch das Flugloch nach aussen transportiert.

Verron (1955) hat mit kleinen Gruppen von Arbeiterinnen (50) gearbeitet, und er vertrat die Ansicht, dass eine Feuchtigkeitsregelung nur durch die Temperaturregelung hervorgerufen wird. Weitere Messungen über die Luftfeuchtigkeit im Beuteninneren verdanken wir WoHLgemuth (1957). Er hat mit einem Elektropsychrometer direkt im Stock gemessen und dabei nur $30 \mathrm{ml}$ Messluft pro Messung gebraucht. Die von ihm ermittelten Werte schwanken im Gegensatz zu BüDEL's Ergebnissen von einer Messung zur anderen häufig um $10 \%$. Die durchschnittliche Feuchte wurde mit $15,7 \mathrm{~g} / \mathrm{m}^{3}$ gemessen.

Krechle (1961) hat einen Zusammenhang zwischen der Konzentration des Honigblaseninhaltes der Bienen und der Wassersammeltätigkeit festgestellt. Bei hohen und steigenden Konzentrationen des Honigblaseninhaltes trinken viele Bienen Wasser.

Simpson (1968) gibt ein Referat über das Kleinklima der Beute. Seine Schlussfolgerung über die Feuchtigkeit in der Traube lautet : « ... ausser der eigentlichen Ventilation gibt es keinen eindeutigen Beweis dafür, dass ein Bienenvolk seine Luftfeuchte aktiv kontrollieren kann; nur das Zusammenziehen der Traube in der Kälte und die Verdunstung an heissen Tagen wirken in Richtung einer Stabilisierung m.

In einem Flugraum hat man die Möglichkeit, die Feuchte der Aussenluft, das Nektarangebot (und dessen $W$ assergehalt) und das Wasserangebot zu kontrollieren. Eine Versuchsreihe über die Relation zwischen : Feuchtigkeit 


\section{der Aussenluft, Feuchtigkeit der Stockluft, Wassersammeltätigkeit, Nektar-} sammeltätigkeit und Bruttätigkeit scheint auf Grund des Erwähnten sinnvoll.

\section{METHODIK}

Die Versuche sind durchgeführt mit einem Bienenvolk (Apis mellifica L.) auf 10 Waben, das bei Beginn der Versuche schon ein Jahr in dem von v. PraAgH (1972) beschriebenen Flugraum stand. Die Feuchtigkeitsregelung des Klimaraumes wird in Abb. l erläutert. Die Luft-

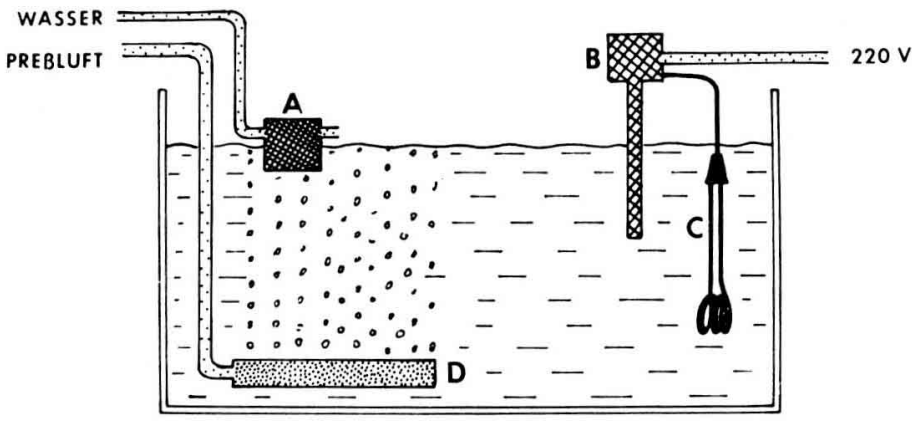

Aвв. 1. - Die Lufteuchtigkeitsregelung.

In das Wasser eines Behälters wird über einen Aquariumstein (D) Luft eingeblasen. Die Luftbläschen werden beim Aufsteigen im Wasser mit Wasserdampf angereichert. Mittels eines Thermostaten (B) und der Heizung (C) wird die Wassertemperatur und damit die Wasserdampfmenge in den Luftbläschen reguliert. Durch einen Niveau-Schalter (A) wird das Wasserniveau im Wasserbehälter aufrechterhalten.

Fic. 1. - La régulation de l'humiditê de l'air. (Explications dans le résumé).

feuchtigkeit und Temperatur der a Aussenluft ${ }^{1}$ ) wurde mittels eines Thermohygrographen registriert. Während der Taupunktmessungen (siehe unten) wurde gleichzeitig die Luftfeuchtigkeit mittels nichtaspirierender Trocken-und Feuchtthermometermessungen bestimmt. Für die Umrechnung haben wir Jecinek's Psychrometer-Tafeln benutzt.

Honigwasser (mit 1 g/l Fumidil B) wurde in Rillenplatten nach ReNNER (1959) angeboten. Der Wassergehalt des Honigwassers wurde refraktometrisch bestimmt. Für Trinkwasser und für eine Kontrolle der Verdunstung des Trinkwassers haben wir zwei Gefässe auf Rillenplatten benutzt (eines vor den Bienen abgeschirmt).

Für Feuchtigkeitsmessungen in einem Bienenvolk wurde ein System zur Bestimmung des Taupunktes in der Wabengasse entwickelt. Ein chromierter Kupferblock $(20 \times 15 \times 6 \mathrm{~mm})$ (Abb. 2) wird mit kaltem Wasser $\left( \pm 2{ }^{\circ} \mathrm{C}\right)$ abgekühlt.

Ein Frequenzmodulator erzeugt eine $1 \mathrm{KHz}$ Lichtfrequenz auf der LED ${ }^{2}$. Das durch einen Spiegel re flektierte Licht wird durch den Phototransistor empfangen und mittels selektiver Verstärkung und Gleichrichtung einem Pegeldetektor zugeleitet. Das Signal des Pegeldetektors und die Spannung des Thermoelements aus Kupfer und Konstantan werden beide gleichzeitig registriert (Kipp-Micrograph BD). In dem Moment, in dem sich Tau auf dem Spiegel absetzt, wird durch den Pegeldetektor ein Signal abgegeben, so dass der Moment der Betauung und die derzeitige Temperatur der Meßstelle festgelegt sind. Bei Vorversuchen hat sich ergeben, dass (wegen des kleinen Volumens) eine Abkühlung mit Äther eine zu unregel-

1. Mit « Aussenluft » wird in dieser Arbeit die Luft im Flugraum bezeichnet; « Innenluft » = Stockluft.

2. LED = Light-Emitting Diode. 


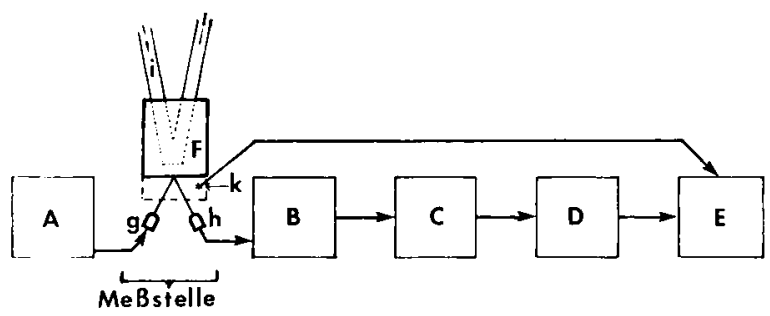

Aвв. 2. - Das Taupunktmeßsystem

A. Frequenzmodulator.

B. Selektiver Verstärker.

C. Gleichrichter.

D. Pegeldetektor.

E. Registriervorrichtung.

F. Kupferblock.

g. LED, wirft ein rotes Lichtsignal $(1 \mathrm{kHz})$ auf die reflektierende Unterseite des Kupferblockes F.

h. Phototransistor, empfängt das reflektierte Licht von g.

i. Kaltes Wasser.

k. Kupfer-Konstantan Thermoelement.

FIG. 2. - Système de mesure du point de rosée.

A. Modulateur de fréquence.

B. Amplificateur sélectif.

C. Redresseur.

D. Indicateur de niveau.

E. Enregistreur.

F. Bloc de cuivre.

g. Diode. Elle envoie un signal lumineux rouge $(1 \mathrm{kHz})$ sur la face inférieure réfléchissante du bloc de cuivre $\mathbf{F}$

h. Eil électronique - reçoit la lumière réfléchit de g.

i. Eau froide.

k. Thermocouple cuivre/constantan.

mässige Abkühlung hervorruft, deshalb haben wir uns für kaltes Wasser entschieden. Abb. 3 gibt eine Registrierung wieder. Bei Punkt $C$ ist die Abkühlung kurzfristig unterbrochen, aber nur scheinbar : Durch Kondensation von Wasserdampf auf der Meßstelle wird Wärme produziert, die die Abkühlung durch das kalte Wasser teilweise kompensiert.

Die Taupunktmessungen wurden mit vier Meßelementen in folgenden Positionen durchgeführt : Wabengasse 4 (zwischen Wabe 3 und 4), Gasse 6, 7 und 10. Zu Beginn der Meßserien waren das Messungen am Brutnestrand, in Brutnestmitte und ausserhalb des Brutnestes (Gasse mit nur verdeckeltem Honig).

\section{ERGEBNISSE}

Dem Rückgang der Bruttätigkeit ging während der Versuchsperiode von zwei Jahren fünfmal eine Senkung der Feuchtigkeit der Aussenluft voran. Der Partialdampfdruck betrug während dieser Periode 13,3 mbar (Standardabweichung 1,95 mbar) und ausserhalb der trockenen Perioden $21,3 \pm 0,7$ mbar. Die Aussentemperatur lag tagsüber bei $23 \pm 0,5^{\circ} \mathrm{C}$ und während der Nacht bei $19 \pm 0,5{ }^{\circ} \mathrm{C}$. Diesen Werten entspricht eine relative Luftfeuchte von $25 \%$ und $40 \%$ bei Brutnesttemperatur (34\%). 


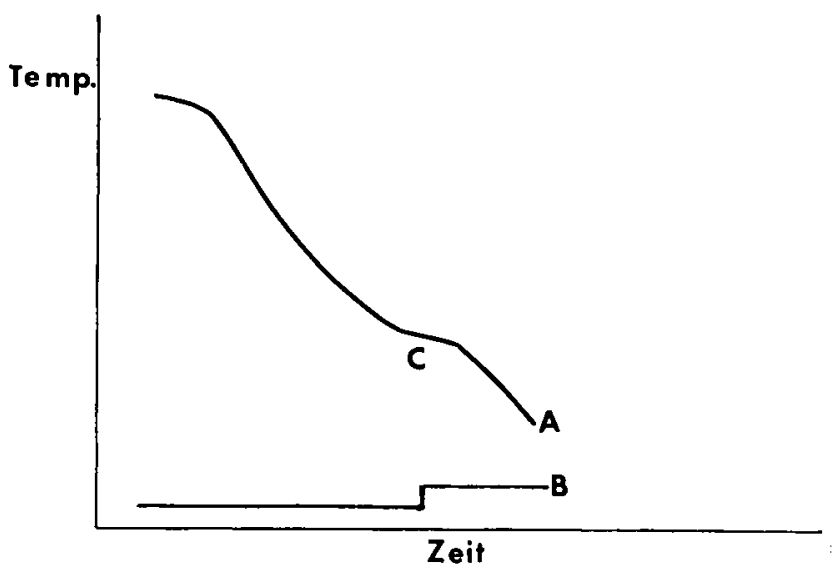

Авв. 3. - Registrationsbeispiel einer Taupunktmessung.

A. Die Temperatur/Zeit-Kurve; bei C setzt sich Tau ab.

B. Das Signal des Pegeldetektors (bei Taubildung ändert sich der Pegel).

Fig. 3. - Exemple d'enregistrement d'un point de rosée.

A. Courbe température/temps; en $\mathrm{C}$ a lieu la condensation.

B. Signal de l'indicateur de niveau (le niveau change au moment de la condensation).

Die Resultate von 56 Tagen Feuchtigkeitsmessung im Stockinneren sind in Abb. 4 dargestellt. Innerhalb dieser 56 Tage haben wir zwei Abnahmen der Bruttätigkeit und der Innenluftfeuchte beobachtet; sie wurden aber durch verschiedene Maßnahmen hervorgerufen.

Die Feuchtigkeit im Stock (innerhalb und ausserhalb des Brutnestes) ist normalerweise korreliert mit der Feuchtigkeit der Aussenluft, obwohl das Stockinnere immer eine höhere Luftfeuchtigkeit hat. Bei einem Rückgang des Wasserdampfes im Stock bis zu einem Wert von 19,5 mbar (Aussenluft 13,3 mbar) geht die Bruttätigkeit zurück. Eine derartige Erniedrigung der Innenluftfeuchtigkeit ist auch zu beobachten, wenn den Bienen reiner Honig als Sammelgut zur Verfügung steht. Zur gleichen Zeit herrscht eine lebhafte Wassersammeltätigkeit im Flugraum, aber die Bruttätigkeit geht trotzdem rasch zurück. Bei trockener Aussenluft hingegen sind die Wassersammlerinnen nicht derart aktiv; die aufgenommene Wassermenge ist normal.

\section{DISKUSSION}

Ein Vergleich zwischen unseren Ergebnissen und der Arbeit von BüDEL ergibt folgendes : 

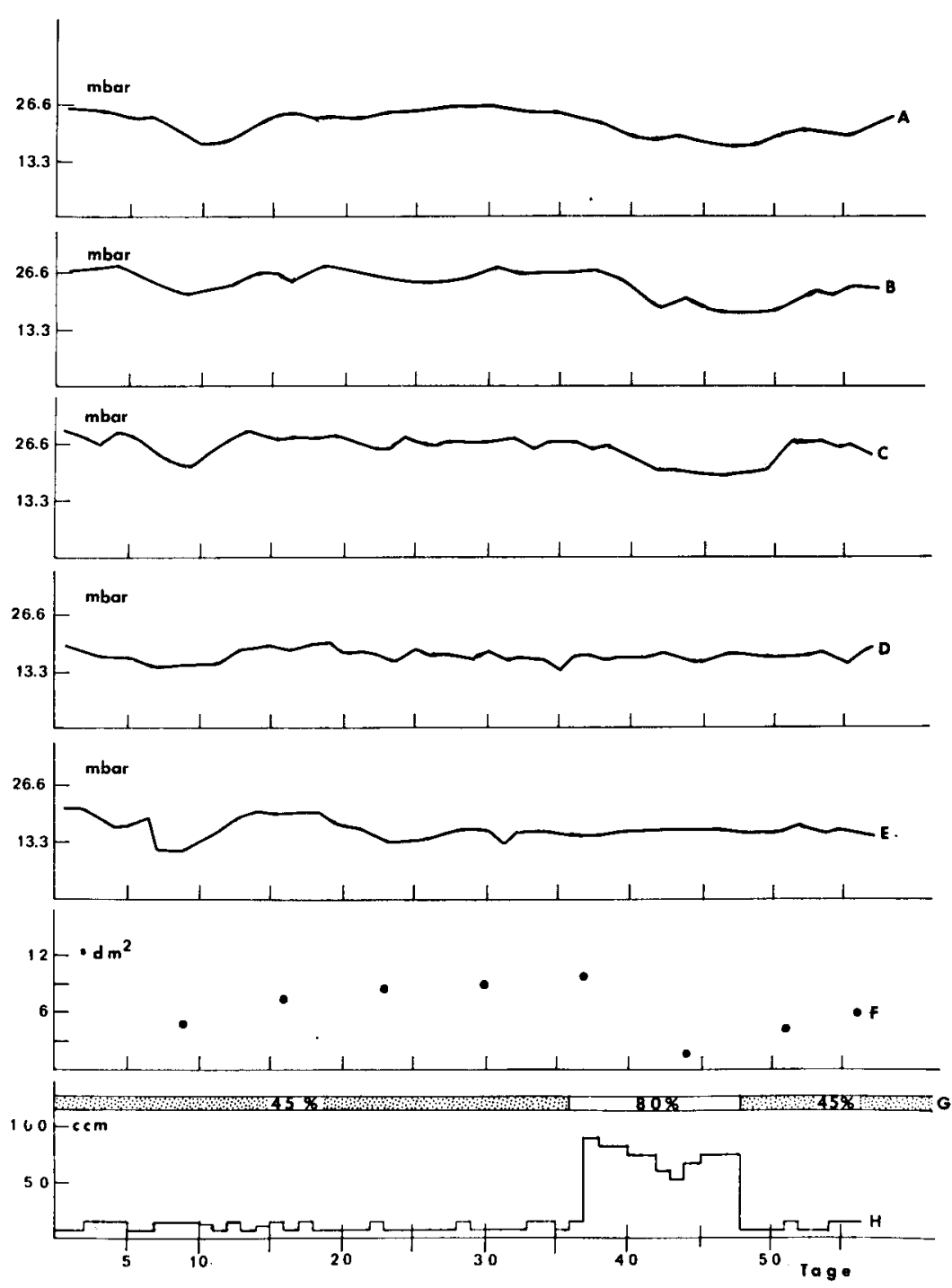

Aвв. 4. - Partialdampfdruck, offene Brut und Sammeltätigkeit.
A. Partialdampfdruckverlauf in Wabengasse $4(\mathrm{~N}=168)$.
$\begin{array}{llll}\text { B. } & \text { " } & * & 6(\mathrm{~N}=168) . \\ \text { C. } & \text { " } & \ll & 7(\mathrm{~N}=168) .\end{array}$
D. $\quad \ll \quad 10(\mathrm{~N}=168)$
E. $\quad$ der Aussenluft $(N=168)$.

F. $\mathrm{dm}^{2}$ offene Brut im Versuchsvolk $(\mathrm{N}=8)$.

G. Zuckerkonzentration im dargebotenen « Nektar ».

H. Pro Tag gesammelte Menge Trinkwasser $(\mathrm{N}=56)$.

Fig. 4. - Pression de vapeur partielle, couvain non operculé et activité de récolte.

A. Pression de vapeur partielle entre les $3^{\mathrm{e}}$ et $4^{\mathrm{e}}$ cadres (situés en bordure du nid à couvain) $(\mathrm{N}=168)$.

B. Pression de vapeur partielle entre les $5^{\mathrm{e}}$ et $6^{\mathrm{e}}$ cadres (situés à l'intérieur du nid à couvain) $(\mathrm{N}=168)$.

C. Pression de vapeur partielle entre les $6^{\mathrm{e}}$ et $7^{\mathrm{e}}$ cadres (situés à l'intérieur du nid à couvain) $(\mathrm{N}=168)$.

D. Pression de vapeur partielle entre les $9^{\mathrm{e}}$ et $10^{\mathrm{e}}$ cadres (situés en dehors du nid à couvain; rayons emplis de miel operculé) $(\mathrm{N}=168)$.

E. Pression de vapeur partielle de l'air extérieur $(\mathrm{N}=168)$.

F. Surface de couvain non operculé en $\mathrm{dm}^{3}(\mathrm{~N}=8)$.

G. Concentration en sucre du « nectar " offert.

$H$. Quantité d'eau récoltée par jour $(\mathrm{N}=168)$. 
Der Mittelwert des Dampfdruckunterschiedes zwischen Innen- und Aussenluft beträgt bei BüDEL $+4,4$ mbar mit einer Standardabweichung (S) von 3,7 mbar; unsere Ergebnisse sind $+6,5$ mbar mit $S=4,4$ mbar. Also etwas höhere Werte, aber die Luft des Brutnestes ist bei beiden Autoren immer feuchter als die Aussenluft. Die von uns beobachteten Werte sind vergleichbar mit den Resultaten von Simpson. Er hat auch Taupunkte bestimmt, aber die Temperatur während der Periode, in der sein Versuchsvolk Brut erzeugte, lag im Brutnest unter $34{ }^{\circ} \mathrm{C}$, was daraufhin deutet, dass das normale Sommerklima sich bei seinem Volk noch nicht eingestellt hatte. Ausserhalb des Brutbezirks ist nicht nur die Temperatur niedriger, sondern auch der Partialdampfdruck. Es bildet sich dort ein Übergang zwischen Brutnest und Aussenluft. Dieser Befund steht im Widerstreit mit der Meinung von BüDEL : « In einer ordnungsgemäss besetzten Beute ist der Wasserdampfgehalt der Luft räumlich im gegebenen Augenblick gleich, zeitlich variabel ». Dazu sind folgende Überlegungen zu berücksichtigen :

BüDEL hat im Brutnest und am Boden des Stockes gemessen und nicht in den Wabengassen neben dem Brutnest. Durch das Absaugen von 0,5 Liter Luft irgendwo aus den Stock findet eine Luftmischung statt und die Luft wird am leichtesten aus dem von wenig Bienen besetzten Stockteilen herausgesaugt. Zu Anfang des Absaugens wird die Luft aus der Nähe des Messpunktes stammen, aber im Moment des Ablesens kann die Luft von irgendeiner anderen Stelle kommen. Infolge der Enge der mit Bienen gefüllten Wabengasse können leicht kleine örtliche Klimaunterschiede auftreten (z.B. im Zusammenhang mit einer erhöhten Stoffwechselaktivität oder mit Nektareindicken oder durch offene Brut). Mit unserer Messmethodik lassen sich solche Unterschiede leicht nachweisen.

Dass die Bienen beim Austrocknen der Stockluft durch die trockene Aussenluft (5.-10. Tag) nicht anfangen, Wasser zu sammeln, sondern dies nur tun bei Fütterung mit reinem Honig (37.-48. Tag), lässt sich aufgrund der Ergebnisse von Lindauer (1954) und Kiechle (1962) erklären. Sie fanden, dass die Zuckerkonzentration in der Honigblase für die Wassersammeltätigkeit wichtig ist. Vom 6. bis zum 10. Tag gab es ein normales Nektarangebot, und die Bienen haben den $45 \%$-igen Honig normal gesammelt und die normale Wassermenge eingetragen. Nach Kroon, v. PraAgh und Velthuis (1974) haben Bienen mit einem Lebensalter von mehr als 16 Tagen unter diesen Umständen eine Zuckerkonzentration von $30 \%$ in ihren Honigblasen. Nach KIECHLE (1962) ist diese Konzentration für massenhaftes Wassersammeln zu niedrig.

Dass es während des 37. bis 48. Tages trotz erhöhter Wassersammeltätigkeit doch zum Austrocknen der Innenluft kommt, lässt sich einfach erklären : Während dieser 11 Tage wurde im Durchschnitt pro Tag $76 \mathrm{~cm}^{3}$ Wasser eingetragen. Während der normalen Sammeltätigkeit wird $560 \mathrm{~cm}^{3} \operatorname{Nektar}(4.5 \%)$ 
gesammelt. Nach Eindicken liefert diese Menge etwa $250 \mathrm{~cm}^{3}$ Wasser pro Tag. Damit ergibt sich, dass ein Bienenvolk in unserem Flugraum nicht in der Lage war, die Feuchtigkeit der Stockluft aktiv auf das für das Erzeugen von grösseren Flächen Brut notwendige Niveau zu bringen. Erhöhte, aber für das Aufrechterhalten der Feuchtigkeit nicht notwendigerweise ausreichende Wassersammeltätigkeit wird nur durch eine hohe Konzentration des Honigblaseneinhaltes, aber nicht durch die niedrige Feuchtigkeit der Stockluft ausgelöst. Bei einem Partialdampfdruck im Brutnest unter $\pm 26,6$ mbar $^{1}$ wird die Brutaktivität eingeschränkt. Infolge des Wasserdampfverlustes an die trockene Aussenluft ist ein Volk in unserem Flugraum nur in der Lage, einen Partialdampfdruckunterschied von $\pm 6,5$ mbar zwischen Innen- und Aussenluft aufrecht zu erhalten. Damit die Feuchtigkeit der Stockluft einen für gute Bruttätigkeit geeigneten Wert hat, sollte der Partialdampfdruck der Aussenluft in unserem Flugraum einen Wert von etwa 15,6 mbar nicht unterschreiten. Bei einer Betrachtung der Klima-Registrierung der Wetterwarte in de Bilt (etwa $6 \mathrm{~km}$ von unserem Flugraum entfernt; K.N.M.I., 1970) ergibt sich, dass der monatliche Mittelwert aus stündlich gemessenen Partialdampfdruckwerten ab September bis Mai diesen Wert (15,6 mbar) unterschreitet. Diese Periode entspricht der Jahreszeit, in der wir immer unsere $W$ asserverdunstungsanlage im Betrieb haben mussten, um normale Bruttätigkeit in unserem Flugraum zu erhalten. Unsere Ergebnisse stehen in Übereinstimmung mit der Schlußfolgerung von Srmpson (1968).

Obwohl Bienen nach Kiechle (1962) in der Lage sind, Feuchtigkeitsunterschiede von $5 \%$ relativer Feuchte ausserhalb des Stockes wahrzunehmen, ist es nach LACHER (1964) ungewiss, ob durch die Feuchtigkeitsrezeptoren der Bienen die relative oder die absolute Feuchte oder auch beide Grössen perzipiert werden können. Unsere Ergebnisse scheinen darauf hinzudeuten, dass die Individuen in einem Volk nicht in der Lage sind, die absolute Feuchtigkeit der Stockluft zu perzipieren, bezw. diese Werte für eine Aktivierung der Wassersammeltätigkeit auszunützen.

Eingegangen im November 1974.

Reçu pour publication en novembre 1974.

\section{RESUMÉ}

On étudie dans une chambre de vol les relations entre l'humidité à l'intérieur et à l'extérieur de la ruche, la miellée et la récolte d'eau par les abeilles. L'humidité à l'intérieur de la chambre de vol est réglée à l'aide d'un bain-marie (Fig. 1). On envoie de l'air comprimé à travers une pierre poreuse (D), située dans le fond du bain-marie; en remontant à la surface les bulles se chargent de vapeur d'eau. La température de l'eau, et avec elle le degré d'hygrométrie des bulles d'air, est réglée à l'aide d'un thermostat (B) et d'une résistance (C). Un indicateur de niveau règle le niveau de l'eau dans le bain-marie.

1. Diesem Wert entspricht eine relative Luftfeuchtigkeit bei $34{ }^{\circ} \mathrm{C}$ von $50 \%$. 
La Fig. 2 décrit le système utilisé pour mesurer les points de rosée entre les rayons de la ruche.

Le modulateur de fréquence $A$ de la diode ( $g$ ) envoie sur un bloc de cuivre chromé une lumière de fréquence $1 \mathrm{kHz}$. La surface de celui-ci la réfléchit sur un œil électronique $(h)$. Ce signal est amplifié sélectivement par $B$, redressé par $C$ et envoyé à l'indicateur de niveau $D$. Le signal et le courant provenant d'un thermocouple cuivre/constantan sont enregistrés tous les deux. Un courant d'eau à $0{ }^{\circ} \mathrm{C}$ refroidit le bloc de cuivre chromé $(12 \times 15 \times 6 \mathrm{~mm})$. Au moment où la rosée se forme sur le miroir, l'intensité lumineuse qui arrive à l'œil électronique diminue (par suite de la diffraction) et l'indicateur de niveau $\mathrm{D}$ déclenche un signal. On détermine ainsi le moment où se forme la rosée et la température à cet instant précis.

La Fig. 3 fournit un exemple d'enregistrement. La courbe A représente le courant du thermocouple, B le signal de l'indicateur de niveau. Au point $\mathrm{C}$ le refroidissement semble interrompu, mais ceci est dû à la chaleur produite par la condensation, qui compense en partie le refroidissement par le courant d'eau.

On observe une diminution de l'hygrométrie de l'air et de la production de couvain lorsque l'hygrométrie à l'extérieur de la ruche est inférieure à 13,3 mbar (humidité relative de $25 \%$ à $34{ }^{\circ} \mathrm{C}$ ). Aucune baisse dans la production de couvain n'est par contre notée lorsque l'hygrométrie de l'air atteint 21,3 mbar (Humidité relative de $40 \%$ à $34{ }^{\circ} \mathrm{C}$ ). La Fig. 4 présente les résultats des mesures d'hygrométrie faites sur 56 jours. Ils permettent de conclure que dans la cage de vol utilisée, une colonie n'est pas capable de régler l'humidité de l'air de la ruche, tel que l'avait supposé Simpson (1968).

Bien que l'air de la ruche renferme plus d'eau que l'air extérieur, on n'a pas observé de différence supérieure à 6,5 mbar. De l'air sec $(19,5 \mathrm{mbar}$, soit $40 \%$ de l'humidité relative) n'induit pas un comportement accru de récolte d'eau ( $5^{\mathrm{e}}$ au $10^{\mathrm{e}}$ jour). Mais lorsqu'on offre du miel pur, une grande quantité d'eau est récoltée ( $37^{\mathrm{e}}$ au $48^{\mathrm{e}}$ jour). Durant les autres jours la quantité d'eau récoltée ne coïncide pas avec la quantité d'eau rapportée à l'intérieur de la ruche $\left(570 \mathrm{~cm}^{3}\right.$ de nectar à $45 \%$ fournissent environ $250 \mathrm{~cm}^{3}$ d'eau; du $37^{\mathrm{e}}$ au $48^{\mathrm{e}}$ jour seulement $76 \mathrm{~cm}^{3}$ d'eau furent récoltés par jour).

\section{LITERATUR}

Bübel A., 1948, Der Wasserdampfhaushalt im Bienenstock. Z. vergl. Physiol., 31, 249-273. HazelhofF E. H., 1941, De Luchtverversching van een Bijenkast gedurende den Zomer. Maandschrift voor Bijenteelt, 44, 1-16.

Jeurnek, 1956, Psychrometer-Tafeln. Ausgabe K.N.M.I., de Bilt, Holland.

Kiechle H., 1962, Die soziale Regulation der Wassersammeltätigkeit im Bienenstaat und deren physiologische Grundlage. Z. vergl. Physiol., 45, 154-192.

K.N.M.I., 1970, Climatological data of Netherlands stations, nr. 3, Frequency tables according to the measurements at De Bilt in the period 1931-1960.

Kroon G. H., van Praagh J. P., Velthuis H. H. W., 1974, Osmotic shock as a prerequisite to pollen digestion in the bee's alimentary tract. J. apic. Res., 13 (3), 177-182.

LACHER V., 1964, Elektrophysiologische Untersuchungen an einzelnen Rezeptoren für Geruch, Kohlendioxid, Luftfeuchtigkeit und Temperatur auf den Antennen der Arbeitsbienen und der Drohne (Apis mellifera L.). Z. vergl. Physiol, 48, 587-623.

Lindauer M., 1954, Temperaturregulierung und Wasserhaushalt im Bienenstaat. Z. vergl. Physiol, 36, 391-4.32.

Renner M., 1959, Über ein weiteres Versetzungsexperiment zur Analyse des Zeitsinnes und der Sonnenorientierung der Honigbiene. Z. vergl. Physiol, 42, 449-483.

Simpson J., 1950, Humidity in the winter cluster of a colony of Honeybees. Bee World, 31 , 41-44.

Srmpson J., 1968, Le microclimat à l'intérieur de la grappe d'abeilles. In : Traité de biologie de l'abeille, tome I, direction R. Chauvin, éd. Masson et Cie, p. 224-234. 
VaN LAERE O., 1963, Ekologische studie van de broednestontwikkeling bij de honingbij (Apis mellifera L.) Proefschrift Rijkslandbouwhogeschool, Gent.

Van Praagh J. P., 1972, Towards a controlled-environment room suitable for normal colony life of honeybees. J. apic. Res., 11, 77-87.

Verron H., 1955, Les réactions des abeilles à l'humidité. Insectes soc., 2, 57-62.

WohlgemUTH R., 1957, Die Temperaturregulation des Bienenvolkes unter regeltheoretischen Gesichtspunkten. Z. vergleich. Physiol., 40, 119-161. 